\title{
The biophysical role of hemodynamics in the pathogenesis of cerebral aneurysm formation and rupture
}

\author{
Sauson Soldozy, BA, Pedro Norat, MD, Mazin Elsarrag, MS, Ajay Chatrath, MS, \\ John S. Costello, BA, Jennifer D. Sokolowski, MD, PhD, Petr Tvrdik, PhD, \\ M. Yashar S. Kalani, MD, PhD, and Min S. Park, MD \\ Department of Neurological Surgery, University of Virginia Health System, Charlottesville, Virginia
}

\begin{abstract}
The pathogenesis of intracranial aneurysms remains complex and multifactorial. While vascular, genetic, and epidemiological factors play a role, nascent aneurysm formation is believed to be induced by hemodynamic forces. Hemodynamic stresses and vascular insults lead to additional aneurysm and vessel remodeling. Advanced imaging techniques allow us to better define the roles of aneurysm and vessel morphology and hemodynamic parameters, such as wall shear stress, oscillatory shear index, and patterns of flow on aneurysm formation, growth, and rupture. While a complete understanding of the interplay between these hemodynamic variables remains elusive, the authors review the efforts that have been made over the past several decades in an attempt to elucidate the physical and biological interactions that govern aneurysm pathophysiology. Furthermore, the current clinical utility of hemodynamics in predicting aneurysm rupture is discussed.
\end{abstract}

https://thejns.org/doi/abs/10.3171/2019.4.FOCUS19232

KEYWORDS cerebral aneurysm; hemodynamics; wall shear stress; computational fluid dynamics; vascular remodeling

I NTRACRANIAL aneurysms (IAs) are acquired outpouchings of arteries that occur in $1 \%-2 \%$ of the population. ${ }^{36}$ Likely as a result of improved imaging modalities, the incidence of unruptured IAs has increased. Despite the estimated $1 \%$ annual risk of rupture, our ability to predict which aneurysms are best managed conservatively versus treated with either open or endovascular techniques is not without controversy. ${ }^{4,65}$ While aneurysm size and location are the main determinants in our decision-making process, there are a multitude of additional factors that are often considered. . $^{17,63}$

Recently, considerable research has focused on the relationship between hemodynamic stressors and aneurysm pathogenesis. From a mathematical and biophysical standpoint, aneurysm morphology and evolution over time is highly complex due to the heterogeneous nature of fluid mechanics. Complicating matters even further is the living nature of blood vessels, i.e., mechanical stimuli are transduced into biological signals, triggering inflammatory cas- cades and, ultimately, a wide range of transcriptional and signaling changes that lead to vascular wall remodeling. The advent of computational and radiographic modeling has allowed for the study of hemodynamics within vessels and aneurysms. For this reason, cerebral aneurysm hemodynamics can serve as a tool in understanding the biophysical pathogenesis of IAs and has been used to evaluate aneurysm rupture risk.

In this article, we review the hemodynamic parameters related to aneurysm formation and rupture and the biophysical pathways that contribute to vascular remodeling. Additionally, we discuss the current state and utility of radiographic and computational modeling in predicting IA rupture in clinical practice.

\section{Aneurysm Morphology}

Aneurysm shape is believed to be an important predictor of subsequent rupture and may be characterized in sev-

ABBREVIATIONS AR = aspect ratio; $B A=$ basilar artery; $C F D=$ computational fluid dynamics; $E C M=$ extracellular matrix; $I A=$ intracranial aneurysm; IEL = internal elastic lamina; IgG = immunoglobulin G; IgM = immunoglobulin M; IL = interleukin; MCP = monocyte chemoattractant protein; MMP = matrix metalloproteinase; MRA = MR angiography; MWSS = maximum WSS; NO = nitric oxide; OSI = oscillatory shear index; SMC = smooth-muscle cell; SR = size ratio; TNF = tumor necrosis factor; VCAM = vascular cell adhesion molecule; VSMC = vascular SMC; WSS = wall shear stress; WSSG = WSS gradient.

SUBMITTED March 2, 2019. ACCEPTED April 18, 2019.

INCLUDE WHEN CITING DOI: 10.3171/2019.4.FOCUS19232. 
eral ways. Ujiie et al ${ }^{61}$ found that an aspect ratio (AR) - the ratio of aneurysm depth to aneurysm neck width-above 1.6 was a reliable predictor of aneurysm rupture. NaderSepahi et al. ${ }^{45}$ also found the AR to be a reliable predictor of rupture, but reported mean ARs of 2.70 and 1.80 for ruptured and unruptured aneurysms, respectively. However, Xiang et al ${ }^{66}$ found that the AR was not significantly different between ruptured and unruptured groups, while Hassan et al. ${ }^{26}$ found sidewall, but not bifurcation, ruptured aneurysms to be strongly associated with AR exceeding 1.6.

The size ratio (SR) of an aneurysm, defined as the ratio between maximum aneurysm height and parent artery diameter, with rupture status has also been explored. Several investigators found strong correlations between greater SR and rupture status. ${ }^{61,68}$ In their systematic review and meta-analysis, Kleinloog et al. ${ }^{33}$ identified irregular aneurysm shape, larger AR, larger SR, higher bottleneck factor (aneurysm width divided by the diameter of the neck), and aneurysm height-to-width ratio as morphological characteristics with strong levels of evidence for increased risk of rupture. Morphological characteristics with moderate levels of evidence for association with rupture included downward/inferior direction of the dome and volume-toostium ratio (ratio of the aneurysm volume to the area of the neck). They suggest that based on the available evidence, the risk factor of irregular shape has high predictive potential and should be considered in risk stratification of aneurysms in clinical practice. ${ }^{44}$

\section{Computational Fluid Dynamics}

Computational fluid dynamics (CFD) creates 3D models of fluid flow from patient CT angiography, MR angiography (MRA), 3D digital subtraction angiography, or 3D rotational angiography. ${ }^{65}$ Aneurysm and vessel characteristics, such as location, size, morphology, AR, and SR, can be used to calculate the wall tension, wall shear stress (WSS), and flow velocity to better estimate aneurysm rupture risk. $3,5,8,9,28,42,54,56,62,65,66$ The results thus far have been quite promising, as CFD has been successfully used to estimate these parameters and predict aneurysm rupture risk in many different studies.

CFD has been used to further investigate the theories of high and low shear stress and aneurysm rupture risk. Castro et al. ${ }^{9}$ created models of 26 anterior communicating artery aneurysms and found significant associations between elevated maximum intraaneurysmal WSS and a clinical history of previous rupture. They observed this association again in later studies examining 42 aneurysms at other locations. ${ }^{7}$ Cebral et al. ${ }^{12}$ analyzed hemodynamic environments in 210 cerebral aneurysms using image-based CFD; they found ruptured aneurysms were more likely to have larger maximum WSS (MWSS). However, other investigations have observed conflicting findings. Xiang et al. ${ }^{66}$ analyzed 119 aneurysms based on 3D angiographic images. They found lower MWSS and WSS in ruptured aneurysms compared to unruptured aneurysms. Shojima et al. ${ }^{55}$ created 20 models of middle cerebral artery vessels with aneurysms from angiography studies, and demonstrated the average WSS of the aneurysm was significantly lower than that of the vessel. Additionally, WSS at the tip of the ruptured aneurysms was markedly low. Other studies also found that aneurysm growth and rupture were more likely to occur in regions of abnormally low WSS, , ,21,32,43,51,58 suggesting that low, rather than high, WSS is associated with aneurysm growth and rupture.

Differences in model assumptions or algorithmic approaches may result in different predicted aneurysm rupture risk scores from the same data..$^{29,30}$ While CFD model predictions have been validated in many different studies, $1,2,4,9$ the results suggest that CFD models will likely become more accurate over time as more data sets are assembled. Complex models integrating multiple different parameters could also further improve CFD model predictions. Further studies in larger patient cohorts are necessary to determine how CFD can be optimized to supplement clinical decision-making as current models are associated with uncertainty and may take hours to days to compute. ${ }^{58}$

\section{Hemodynamic Parameters Aneurysm Initiation: WSS}

The effect of arterial WSS on aneurysm pathogenesis has been the subject of extensive study. Shear stress is composed of the tangential, frictional forces that exist between blood and the stationary vessel wall (Fig. 1). ${ }^{53}$ The relative difference in velocity between two parallel objects creates a shear stress. The shear stress becomes proportional to the velocity gradients between the two objects. As a derivative, the WSS gradient (WSSG) is defined as the spatial derivative of WSS along the direction of flow. It can be thought of as the change in WSS along the length of the vessel. ${ }^{15}$ In response to physiological levels of shear stress, endothelial cells assume a quiescent and atheroprotective gene expression profile characterized by resistance to inflammation, apoptosis, and oxidative stress. Low levels of shear stress promote atherosclerosis by inducing inflammation and an atherogenic phenotype. ${ }^{37,48,59,63}$ When discussing the pathophysiology of IAs, it is important to distinguish aneurysm initiation and growth as two separate entities with distinct hemodynamic and inflammatory mechanisms involved.

WSS is an important parameter in animal aneurysm formation models. Gao et al. ${ }^{22}$ identified high WSS as an important factor in aneurysm formation in a rabbit model. Dramatic increases in basilar artery (BA) flow rate (105\%900\%) and nascent aneurysm formation at the BA bifurcation were found following common carotid artery ligation. Histologically, the nascent aneurysms were characterized by a bulging and thinned tunica media as well as loss of the internal elastic lamina (IEL). The extent of aneurysm sac formation was related to the degree of BA flow-rate increase. High WSS and WSSG were implicated in vessel remodeling and eventual aneurysm formation, with the authors theorizing that endothelial cells at bifurcation apices became dysfunctional under sustained abnormal hemodynamic conditions. Other studies utilizing animal models reported similar findings. ${ }^{1,14,23,35,39,41,54}$ A systematic review and meta-analysis conducted by $\mathrm{Can}$ and $\mathrm{Du}^{6}$ found a high positive correlation between elevated WSS and location of aneurysm formation. While a significant association of 
high WSSG with aneurysm formation was not found, they found that sidewall and bifurcation aneurysms were impacted differently by these hemodynamic factors.

\section{Enlargement and Rupture: Low- and High-Stress Theories}

Although aneurysm formation has been clearly linked to regions of high WSS, the impact of the hemodynamic environment on aneurysm enlargement and rupture is less certain. Conflicting studies have found both high and low WSS to be related to aneurysm growth and eventual rupture.

This apparent conflict has led to the creation of two schools of thought: the high- and low-stress theories. In both theories, the hemodynamic environment within the aneurysm interacts with the cellular elements of the aneurysm wall resulting in further weakening. The differences, however, revolve around the mechanisms responsible for the wall weakening. ${ }^{54}$ The high-stress theory asserts that elevation of maximum WSS causes endothelial injury and initiates processes of wall remodeling and degeneration. Vascular endothelium exposed to elevated MWSS overexpresses nitric oxide (NO), leading to abnormally low arterial tone and apoptosis of wall-embedded smooth-muscle cells (SMCs). ${ }^{54}$

Conversely, the low-stress theory states that stagnation of blood within the aneurysm leads to red blood cell aggregation and buildup of platelets and leukocytes. These changes cause intimal damage and inflammatory cell infiltration of the vessel wall, leading to wall degeneration and a reduced ability to withstand physiological hemodynamic forces. ${ }^{54}$

Meng et al. ${ }^{40}$ proposed that low WSS (as well as high oscillatory shear index [OSI]) leads to a proinflammatory endothelial cell phenotype and increased inflammatory cell infiltration, matrix metalloproteinase (MMP) production, SMC proliferation and migration, and thrombus formation. In contrast, high WSS combined with a positive WSSG resulted in endothelial cell damage and turnover, MMP production by mural cells, extracellular matrix (ECM) degradation, thinning of the media, and apoptosis of SMCs and fibroblasts. They classified these distinct responses as mural cell-mediated and inflammatory cell-mediated destructive remodeling pathways, each of which may drive aneurysm rupture under different hemodynamic conditions and in different aneurysm phenotypes. The mural cell-mediated pathway associated with high WSS could be associated with the growth and rupture of small or secondary bleb aneurysm phenotypes, while the inflammatory-mediated pathway may be responsible for the growth and rupture of large, atherosclerotic, and thrombotic IA phenotypes.

In contrast to Meng et al. ${ }^{40}$ a study comparing in vivo flow conditions with the histology of human IAs suggested the exact opposite association between WSS and aneurysm wall degeneration and rupture. ${ }^{10}$ Using the degree of CD45-positive and CD68-positive cells as a marker of leukocyte and macrophage expression, respectively, Cebral et al. ${ }^{10}$ found that conditions of high WSS were associated with higher levels of inflammation. However, low WSS conditions displayed negative CD45 immunostaining, and were instead associated with loss of mural cells. Immuno-

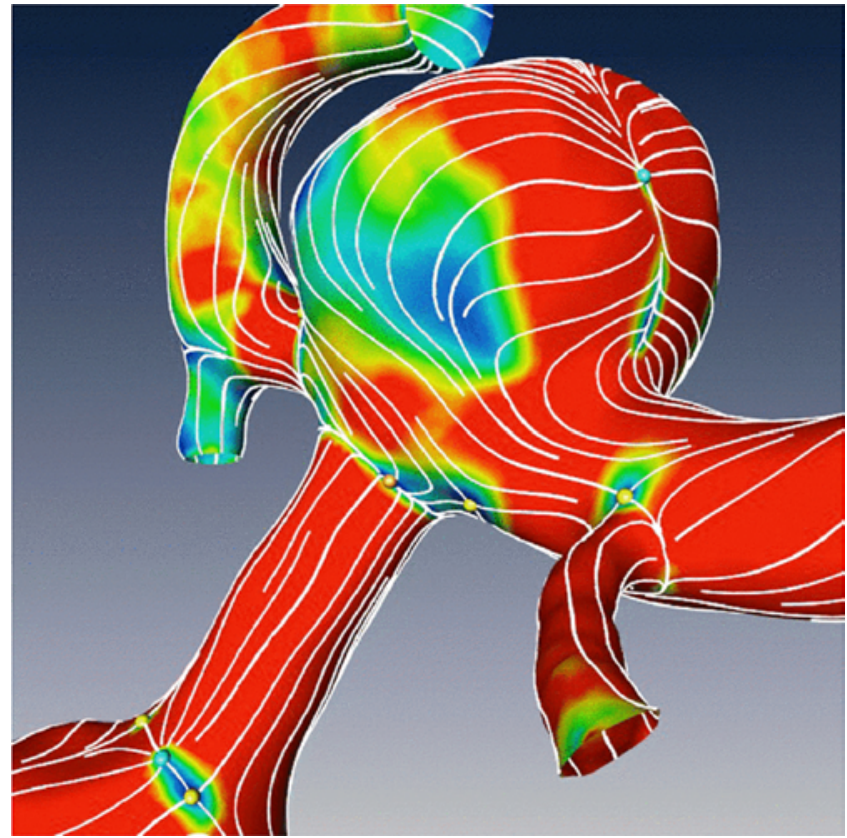

FIG. 1. CFD simulation of blood flow in a reconstructed cerebral aneurysm. Pressure distribution is color-coded with overlying WSS streamlines, depicting overall flow behavior and local topological details. Critical points are depicted by the small spheres. From: Rosanwo O, Petz C, Prohaska S, Hege HC, Hotz I: Dual streamline seeding, in Eades P, Ertl T, Shen HW (eds): IEEE Pacific Visualization Symposium 2009. Piscataway, NJ: IEEE, 2009, pp 9-16. (c) 2019 IEEE. Reprinted with permission from IEEE Proceedings.

histochemistry series have demonstrated higher degrees of inflammation in ruptured aneurysms compared with unruptured aneurysms. This inflammatory infiltrate may be present in vessel walls prior to rupture..$^{19}$ It remains unclear whether inflammation is the cause of wall degeneration and rupture or if it is a reaction to the cause of degenerative remodeling that ultimately leads to rupture..$^{10}$

Despite not knowing the exact mechanism involved, a recent report by Cebral et al. ${ }^{11}$ supports the theory that different hemodynamic conditions are associated with different aneurysm phenotypes. Compared to normal-appearing regions, a significantly lower average WSS was found in atherosclerotic, hyperplastic, and rupture wall types. In thin-wall types, no significant difference in average WSS was found when compared to normal regions, although it was found to be higher than hyperplastic and ruptured regions. Thin-wall regions are more likely aligned in parallel with the flow stream of the aneurysm and experience higher flow rates and have higher average WSS. This is contrasted with thicker wall types (atherosclerotic and hyperplastic), which are perpendicular to the flow stream and are more likely to be associated with low WSS. ${ }^{11}$

\section{Oscillatory Shear Index}

The OSI is a variable that provides a measure of the changes in the direction of the shear forces during the cardiac cycle. ${ }^{13}$ Shearing forces are tangential forces that are parallel to the direction of blood flow. Areas where the direction of the WSS vector changes frequency through- 

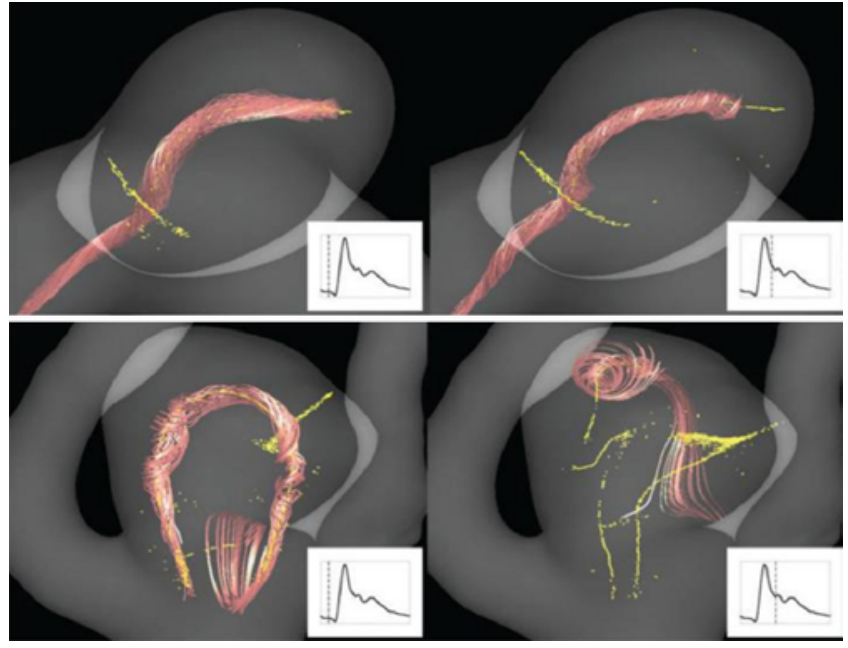

FIG. 2. Examples of stable (upper) and unstable (lower) temporal blood flows. Vortex core lines (yellow) and neighboring streamline trajectories (red) are used to visualize the spatial structure of the flow at two instants during the cardiac cycle. Given its consistency and retained spatial structure over the cardiac cycle, the upper aneurysm would be classified as having a low OSI. The lower aneurysm, with large fluctuations in its spatial structure, would have a high OSI. From: Byrne G, Mut F, Cebral $\mathrm{J}$ : Quantifying the large-scale hemodynamics of intracranial aneurysms. AJNR Am J Neuroradiol 35:333-338, 2014. Published with permission.

out the cardiac cycle (e.g., areas of flow recirculation or reversed flow) will have high OSI values. ${ }^{24}$ OSI measures temporal, rather than spatial, variation in flow direction. If the direction of flow at a given point is maintained throughout the cardiac cycle in an aneurysm with several different directions of flow, the OSI will be low at that point. Temporal variations in blood flow can be illustrated by the vortices of blood flow that are created and disappear throughout the cardiac cycle (Fig. 2). ${ }^{5}$

Given the importance of aneurysm geometry and parent vessel characteristics to intraaneurysmal hemodynamics ${ }^{60}$ OSI may reflect aneurysm and parent-vessel morphological features. Low WSS is often accompanied by oscillatory flow, which may enhance atherogenesis, promote inflammation, and lead to intimal wall thickening. ${ }^{54,66}$ Although a number of studies have identified high OSI in ruptured aneurysms, $\mathrm{Can}$ and $\mathrm{Du}^{6}$ found that ruptured aneurysms did not have significantly different pooled OSI compared with unruptured aneurysms in a systematic review and meta-analysis. While Zhou et al. ${ }^{69}$ found a higher OSI in ruptured aneurysms, this finding did not reach statistical significance in their review. Oscillatory flow may be a contributing factor to adverse vascular remodeling that precedes aneurysm rupture, but its exact role in predicting aneurysm rupture remains uncertain.

\section{Flow Patterns}

Flow patterns within the aneurysm sac may also influence risk of rupture. Blood flow in singular and consistent patterns would be considered simple, while complex flow patterns are characterized by multiple areas of recirculation that may persist or temporarily exist throughout the cardiac cycle. ${ }^{54}$ Byrne et al. ${ }^{5}$ found that ruptured aneu-

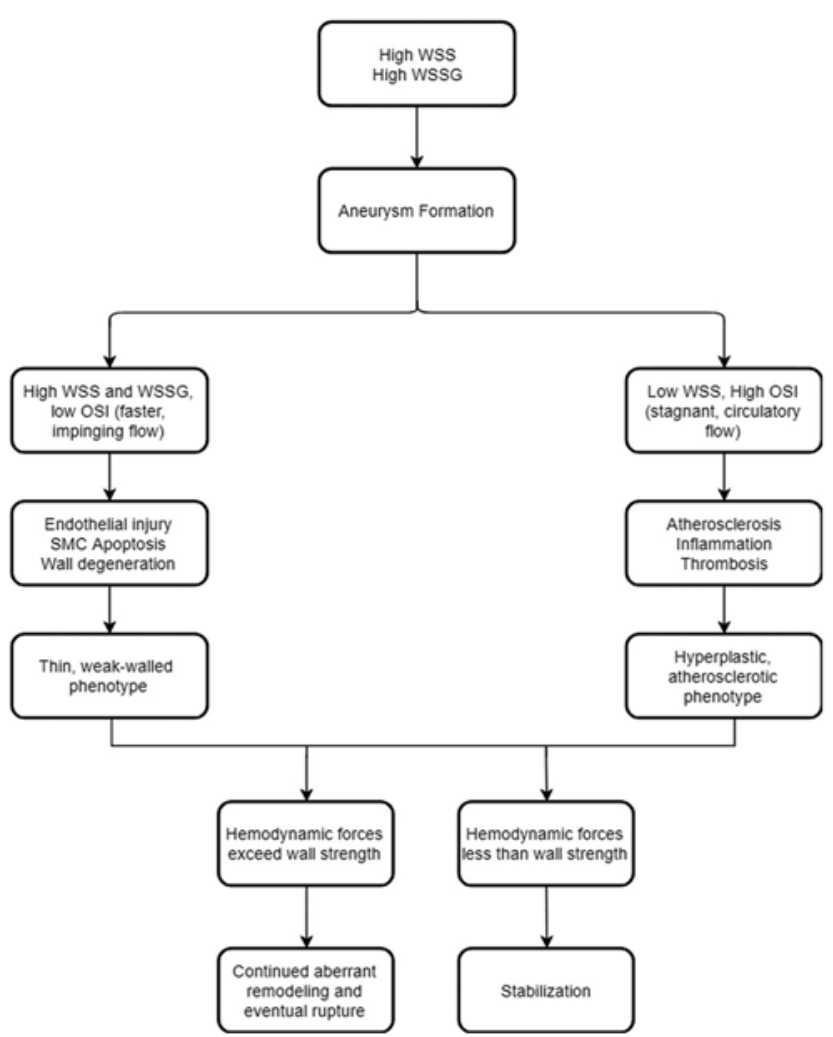

FIG. 3. Flowchart outlining the different hemodynamic factors that lead to aneurysm formation and rupture. Aneurysm formation occurs in a manner distinct from aneurysm rupture, with two proposed mechanisms for explaining vessel wall remodeling and eventual rupture. In the high-WSS theory, it is believed that SMC apoptosis and endothelial degeneration lead to a thin-walled phenotype. In contrast, the low-WSS theory suggests an inflammatory mechanism involving hyperplasia and atherosclerosis. The extent of continued hemodynamic instability with respect to wall strength ultimately leads to either aneurysm rupture or stabilization.

rysms had more complex and unstable flow patterns than unruptured aneurysms. Additionally, spatial complexity was more strongly associated with rupture than temporal stability. Xiang et al. ${ }^{67}$ found that most ruptured aneurysms exhibited complex flow with multiple vortices, while most unruptured aneurysms demonstrated simple flow patterns with a single vortex. Other reports have also associated rupture with complex flow patterns..$^{21}$ However, Kleinloog et al. ${ }^{33}$ concluded that complex flow patterns had a low level of evidence for association with rupture in their systematic review. When the vascular wall can no longer tolerate hemodynamic stress, it ultimately leads to rupture (Fig. 3).

\section{Hemodynamics and Vascular Remodeling}

Abnormal hemodynamics serves as a trigger for vascular modeling and the biochemical mechanisms that persist following the inciting stressor. Arterial remodeling is an adaptive process that attempts to return high or low WSS back to a physiological baseline of 15-20 dynes/ $\mathrm{cm}^{2} .^{25}$ Vascular remodeling is characterized by vascular SMC (VSMC) apoptosis and migration, degradation of the ECM, and inflammation causing local dilation and thin- 


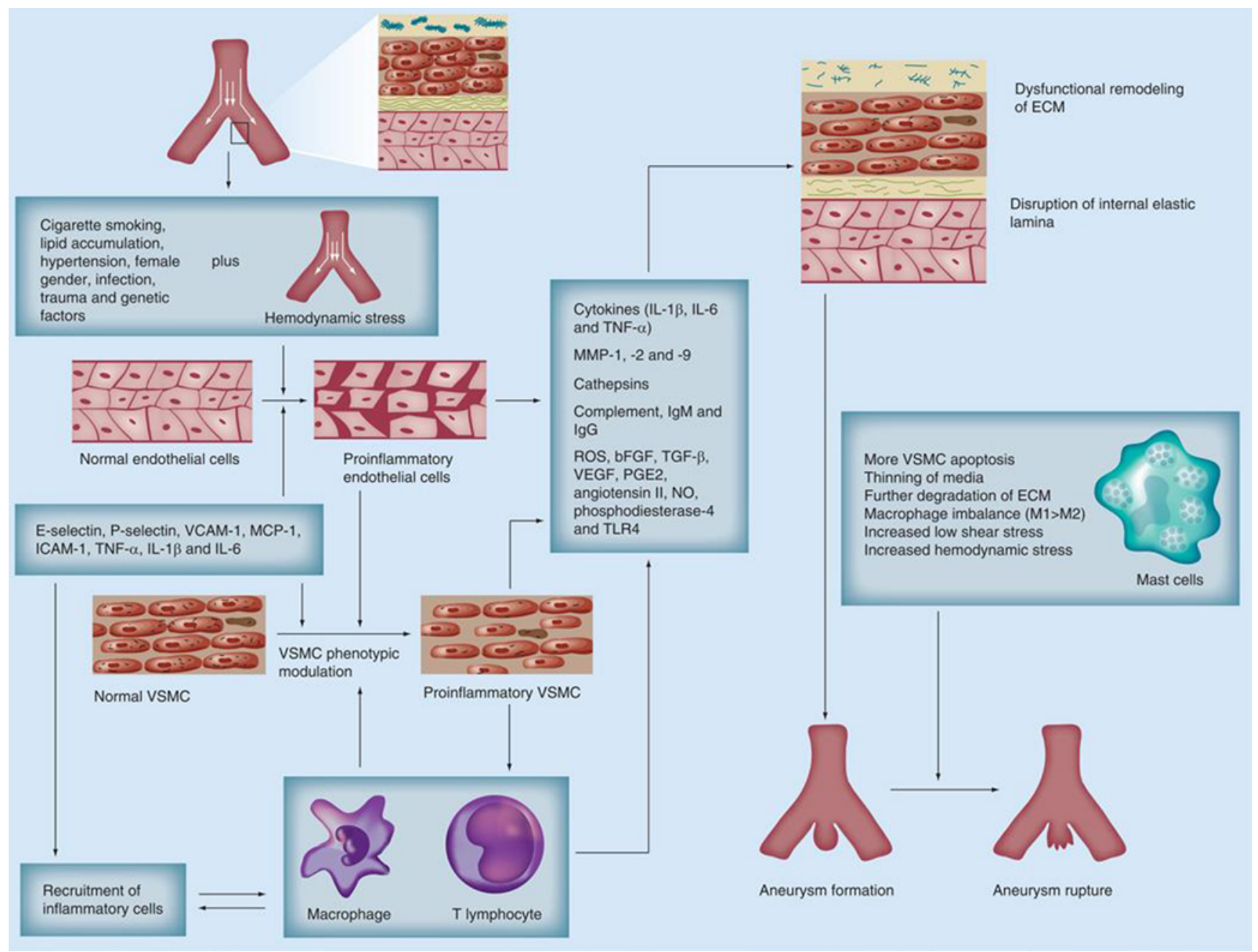

FIG. 4. Schematic outlining the inflammatory mediators involved in aneurysm formation and progression to rupture. Following hemodynamic stress, the vascular endothelium becomes predisposed to remodeling and degeneration, mediated by macrophages, T lymphocytes, and other inflammatory cells. VSMCs undergo phenotypic modulation to a proinflammatory state, the IEL is disrupted, and the ECM is degraded, leading to aneurysm formation and eventual rupture. bFGF = basic fibroblast growth factor; ICAM = intercellular adhesion molecule; $\mathrm{M} 1$ = proinflammatory macrophage; $\mathrm{M} 2$ = antiinflammatory macrophage; NK = natural killer cell; PGE = prostaglandin E; ROS = reactive oxygen species; TGF = transforming growth factor; TLR = toll-like receptor; VEGF = vascular endothelial growth factor. Republished with permission of Hudson JS, Hoyne DS, Hasan DM: Inflammation and human cerebral aneurysms: current and future treatment prospects. Future Neurol 8:10.2217/fnl.13.40, 2013; permission conveyed through Copyright Clearance Center, Inc.

ning of the vessel wall (Fig. 4). ${ }^{28,52}$ Additionally, the biological interactions of flow and vessel wall remodeling that initiate aneurysm formation is a distinct process from the growth and rupture of an already formed aneurysm.

In the presence of hemodynamic stressors, flow-dependent NO release inhibits the proliferation of VSMCs and can initiate apoptosis by activating caspase 3 . This results in pyknosis, karyorrhexis, and cell death. In addition, physical stretch on the vessel wall can induce apoptosis by activation of the tumor suppressor p53. Primarily found in the medial layer, VSMC migration is a normal response in the setting of vascular injury. Hemodynamic stimuli may alter migratory signals such as amines, peptide growth factors, cytokines, and ECM components, such that migration contributes to vessel wall thinning. ${ }^{52}$ Migration of VSMCs is in the direction of the intima, with a subsequent phenotypic reprogramming mediated by tumor necrosis factor-alpha (TNF- $\alpha$ ) and Kruppel-like transcription factor 4. VSMCs gain the ability to express genes related to upregulation of proinflammatory molecules including MMPs, monocyte chemoattractant protein-1 (MCP-1), vascular cell adhesion molecule-1 (VCAM-1), and interleukin (IL). ${ }^{56}$ Within the intimal layer, these cells can proliferate and synthesize new matrix and fibrous tissue, resulting in the process known as intimal hyperplasia. Normally a part of the wound-healing process, decellularized areas characterized by loss of mural cells, matrix breakdown, and hyalinization resembling fibrinoid necrosis have been detected in rupture-prone aneurysms. ${ }^{19}$

The disappearance of the IEL is among the first histological changes observed during aneurysmal growth, leaving the adventitia as the only layer resisting transmural 
blood pressure. ECM proteins continue to become compromised with MMPs degrading structural proteins such as collagens, elastin, proteoglycans, laminin, and fibronectin. MMP activity is further upregulated by flow-dependent NO release through posttranslational modification. ${ }^{52}$ Specifically, overexpression of MMP-1, -2 , and -9 has been identified in aneurysm walls with MMP-2 and -9 being found at higher levels in ruptured aneurysms. ${ }^{31}$ Chronic proteolytic injury by collagenases is further enhanced by the downregulation of antiapoptotic genes. ${ }^{19}$

Monocytes are among the first cell types to respond to hemodynamic endothelial damage, infiltrating the site of injury and differentiating into macrophages that secrete cytokines and proteinases. MCP-1, TNF- $\alpha$, and stromal cell-derived factor-1 (SDF-1; also known as CXCL12) are among the cytokines released by macrophages. SDF-1/ CXCL12 functions to recruit endothelial progenitor cells and induce angiogenesis and further inflammatory cell migration and infiltration. ${ }^{27}$ Macrophages also contribute to further ECM degradation by secreting MMPs. CD163positive, the primary macrophage found in IAs, expresses a specific receptor that is a member of the scavenger receptor cysteine-rich superfamily. ${ }^{16}$ These macrophages function to bind hemoglobin:haptoglobin complexes and are triggered by high levels of oxidative stress contributing to their role in IA wall degeneration and rupture. ${ }^{16,19}$ Macrophage infiltration has also been correlated with intracellular lipid accumulation and apolipoproteins, including ApoA- $\mathrm{I}^{50}$ and ApoB100. ${ }^{18}$

A difference in atherosclerotic lesions is evident between small and large aneurysms. In small aneurysms, atherosclerotic lesions are characterized by diffuse intimal thickening with predominantly VSMCs and minimal macrophages and lymphocytes. In contrast, larger aneurysms have more advanced atherosclerotic lesions with macrophages as the primary cellular infiltrate. ${ }^{34}$ Interestingly, Frösen et al..$^{18}$ noted that acquired antibodies against oxidized lipids within the vasculature served a protective role, with lower levels of oxidized low-density lipoprotein-reactive immunoglobulin $\mathrm{G}(\mathrm{IgG})$ antibodies associated with a history of IA rupture.

Humoral immune response has also been shown to be active in aneurysm walls in the form of complement component and antibody (immunoglobulin $\mathrm{M}[\operatorname{IgM}]$ and $\mathrm{IgG}$ ) deposition. ${ }^{19}$ Significant amounts of C3d accumulation have been observed in aneurysms, suggesting that a more chronic inflammatory state may be involved. Activation of the classic pathway with an alternative pathway amplification has also been implicated, causing the release of chemokines that further facilitate the recruitment of macrophages and T cells. Membrane attack complexes are rarely observed on the cell surface; instead, they are found within the matrix and cellular debris of degraded regions. This suggests complement activation may occur as a reaction to necrosis, rather than the cause of it..$^{19}$

\section{Predicting Aneurysm Rupture With Hemodynamics \\ MRI}

Advances in MRI development and technology show significant promise for predicting aneurysm rupture risk. Four-dimensional flow MRI, also known as 3D time-resolved phase-contrast MRI, quantifies blood flow in three dimensions and can be used to estimate WSS, SR, and inflow hemodynamics much faster than traditional CFD, although 4D MRI often underestimates WSS. ${ }^{21,57}$ Contrast permeability across aneurysm walls as measured by dynamic contrast-enhanced MRI has been found to be predictive of aneurysm rupture risk independent of aneurysm size. ${ }^{62}$ Seven-tesla MRI has been used to measure wall thickness, which was found to be inversely related to WSS. ${ }^{2}$

The presence of atherosclerotic plaques in IAs has been extensively studied. ${ }^{18,34,50}$ Hybrid of opposite-contrast MRA is a combination of time-of-flight and flow-sensitive black-blood MRA that can be used to visualize atherosclerotic plaques along aneurysm walls. ${ }^{38}$ Even in the absence of visible atherosclerotic changes such as fatty streaks, the accumulation of oxidized lipids is associated with wall inflammation. ${ }^{34}$

Wall enhancement and microhemorrhage identification have also been explored as a tool for predicting aneurysm rupture. ${ }^{49}$ MRI with 3D turbo spin-echo sequences with motion-sensitized driven equilibrium can be used to detect aneurysm wall enhancement. While ruptured aneurysms are more often associated with aneurysm wall enhancement, it is still unclear whether aneurysm wall enhancement in unruptured aneurysms is suggestive of increased rupture risk. ${ }^{46}$ Circumferential aneurysm wall enhancement detected by 3T gadolinium-enhanced high-resolution MRI has been found to be associated with symptomatic aneurysms compared to asymptomatic aneurysms, although its ability to predict aneurysm rupture has not yet been studied. ${ }^{20}$

Quantitative susceptibility mapping localized paramagnetic metals such as ferric iron and was used to detect microhemorrhages in a case report. ${ }^{47} \mathrm{~T} 2$-weighted gradientrecalled echo sequence MRI was used to detect cerebral microbleeds in a large study of 1847 patients and found to be associated with an increased risk of aneurysm rupture. ${ }^{68}$ While further research is needed to evaluate the usefulness of these methodologies in clinical practice, several hemodynamic parameters may predict aneurysm formation or rupture, summarized in Table 1.

\section{Conclusions}

As imaging studies and computational modeling continue to improve, understanding of the hemodynamic role in cerebral aneurysm formation, growth, and rupture will increase. While WSS is implicated in aneurysm initiation, its role in aneurysm growth and rupture remains controversial, with studies showing contrasting results. Other hemodynamic parameters may be involved in aneurysm pathogenesis, although they are much less studied than WSS. It appears that the cumulative effect of these hemodynamic stressors triggers an array of inflammatory and signaling cascades that ultimately results in vessel wall thinning, dilation, and rupture. With respect to imaging, current advances in MRI techniques and CFD provide a promising modality for predicting aneurysm rupture in patients, although a more integrative approach that incorpo- 
TABLE 1. Summary of flow and imaging parameters and their associated risk with aneurysm rupture or formation

\begin{tabular}{lll}
\hline \multicolumn{1}{c}{ Parameter } & Association & \multicolumn{1}{c}{ Risk } \\
\hline $\begin{array}{l}\text { Aneurysm wall contrast perme- } \\
\text { ability }\end{array}$ & Aneurysm rupture & Increased \\
\hline Aneurysm wall enhancement & Aneurysm rupture & $\begin{array}{c}\text { Possibly } \\
\text { increased }\end{array}$ \\
\hline AR & Aneurysm rupture & Unclear \\
\hline Atherosclerotic plaques & Aneurysm rupture & Unclear \\
\hline Bottleneck factor & Aneurysm rupture & Increased \\
\hline Complex flow patterns & Aneurysm rupture & Increased \\
\hline Height-to-width ratio & Aneurysm rupture & Increased \\
\hline Irregular aneurysm shape & Aneurysm rupture & Increased \\
\hline Microhemorrhage identification & Aneurysm rupture & Increased \\
\hline OSI & Aneurysm rupture & Unclear \\
\hline SR & Aneurysm rupture & Increased \\
\hline WSS & Aneurysm rupture & Unclear \\
\hline
\end{tabular}

rates vascular, genetic, and epidemiological risk factors of aneurysm rupture is needed to be a truly predictive model with clinical utility.

\section{References}

1. Alfano JM, Kolega J, Natarajan SK, Xiang J, Paluch RA, Levy EI, et al: Intracranial aneurysms occur more frequently at bifurcation sites that typically experience higher hemodynamic stresses. Neurosurgery 73:497-505, 2013

2. Blankena R, Kleinloog R, Verweij BH, van Ooij P, Ten Haken $\mathrm{B}$, Luijten PR, et al: Thinner regions of intracranial aneurysm wall correlate with regions of higher wall shear stress: a 7T MRI study. AJNR Am J Neuroradiol 37:1310-1317, 2016

3. Boussel L, Rayz V, McCulloch C, Martin A, Acevedo-Bolton G, Lawton M, et al: Aneurysm growth occurs at region of low wall shear stress: patient-specific correlation of hemodynamics and growth in a longitudinal study. Stroke 39:29973002, 2008

4. Brown RD Jr, Broderick JP: Unruptured intracranial aneurysms: epidemiology, natural history, management options, and familial screening. Lancet Neurol 13:393-404, 2014

5. Byrne G, Mut F, Cebral J: Quantifying the large-scale hemodynamics of intracranial aneurysms. AJNR Am J Neuroradiol 35:333-338, 2014

6. Can A, Du R: Association of hemodynamic factors with intracranial aneurysm formation and rupture: systematic review and meta-analysis. Neurosurgery 78:510-520, 2016

7. Castro M, Putman C, Radaelli A, Frangi A, Cebral J: Hemodynamics and rupture of terminal cerebral aneurysms. Acad Radiol 16:1201-1207, 2009

8. Castro MA, Putman CM, Cebral JR: Patient-specific computational fluid dynamics modeling of anterior communicating artery aneurysms: a study of the sensitivity of intra-aneurysmal flow patterns to flow conditions in the carotid arteries. AJNR Am J Neuroradiol 27:2061-2068, 2006

9. Castro MA, Putman CM, Sheridan MJ, Cebral JR: Hemodynamic patterns of anterior communicating artery aneurysms: a possible association with rupture. AJNR Am J Neuroradiol 30:297-302, 2009

10. Cebral J, Ollikainen E, Chung BJ, Mut F, Sippola V, Jahromi $\mathrm{BR}$, et al: Flow conditions in the intracranial aneurysm lumen are associated with inflammation and degenerative changes of the aneurysm wall. AJNR Am J Neuroradiol 38:119-126, 2017

11. Cebral JR, Detmer F, Chung BJ, Choque-Velasquez J, Rezai $\mathrm{B}$, Lehto $\mathrm{H}$, et al: Local hemodynamic conditions associated with focal changes in the intracranial aneurysm wall. AJNR Am J Neuroradiol 40:510-516, 2019

12. Cebral JR, Mut F, Weir J, Putman C: Quantitative characterization of the hemodynamic environment in ruptured and unruptured brain aneurysms. AJNR Am J Neuroradiol 32:145-151, 2011

13. Cebral JR, Yim PJ, Löhner R, Soto O, Choyke PL: Blood flow modeling in carotid arteries with computational fluid dynamics and MR imaging. Acad Radiol 9:1286-1299, 2002

14. Chen H, Selimovic A, Thompson H, Chiarini A, Penrose $\mathrm{J}$, Ventikos Y, et al: Investigating the influence of haemodynamic stimuli on intracranial aneurysm inception. Ann Biomed Eng 41:1492-1504, 2013

15. Dolan JM, Kolega J, Meng H: High wall shear stress and spatial gradients in vascular pathology: a review. Ann Biomed Eng 41:1411-1427, 2013

16. Fabriek BO, Dijkstra CD, van den Berg TK: The macrophage scavenger receptor CD163. Immunobiology 210:153-160, 2005

17. Feigin VL, Rinkel GJE, Lawes CMM, Algra A, Bennett DA, van Gijn J, et al: Risk factors for subarachnoid hemorrhage: an updated systematic review of epidemiological studies. Stroke 36:2773-2780, 2005

18. Frösen J, Tulamo R, Heikura T, Sammalkorpi S, Niemelä M, Hernesniemi J, et al: Lipid accumulation, lipid oxidation, and low plasma levels of acquired antibodies against oxidized lipids associate with degeneration and rupture of the intracranial aneurysm wall. Acta Neuropathol Commun 1:71, 2013

19. Frösen J, Tulamo R, Paetau A, Laaksamo E, Korja M, Laakso A, et al: Saccular intracranial aneurysm: pathology and mechanisms. Acta Neuropathol 123:773-786, 2012

20. Fu Q, Guan S, Liu C, Wang K, Cheng J: Clinical significance of circumferential aneurysmal wall enhancement in symptomatic patients with unruptured intracranial aneurysms: a high-resolution MRI study. Clin Neuroradiol 28:509-514, 2018

21. Futami K, Nambu I, Kitabayashi T, Sano H, Misaki K, Uchiyama N, et al: Inflow hemodynamics evaluated by using fourdimensional flow magnetic resonance imaging and the size ratio of unruptured cerebral aneurysms. Neuroradiology 59:411-418, 2017

22. Gao L, Hoi Y, Swartz DD, Kolega J, Siddiqui A, Meng H: Nascent aneurysm formation at the basilar terminus induced by hemodynamics. Stroke 39:2085-2090, 2008

23. Geers AJ, Morales HG, Larrabide I, Butakoff C, Bijlenga P, Frangi AF: Wall shear stress at the initiation site of cerebral aneurysms. Biomech Model Mechanobiol 16:97-115, 2017

24. Glor FP, Long Q, Hughes AD, Augst AD, Ariff B, Thom SAM, et al: Reproducibility study of magnetic resonance image-based computational fluid dynamics prediction of carotid bifurcation flow. Ann Biomed Eng 31:142-151, 2003

25. Hashimoto T, Meng H, Young WL: Intracranial aneurysms: links among inflammation, hemodynamics and vascular remodeling. Neurol Res 28:372-380, 2006

26. Hassan T, Timofeev EV, Saito T, Shimizu H, Ezura M, Matsumoto Y, et al: A proposed parent vessel geometry-based categorization of saccular intracranial aneurysms: computational flow dynamics analysis of the risk factors for lesion rupture. J Neurosurg 103:662-680, 2005

27. Hoh BL, Hosaka K, Downes DP, Nowicki KW, Wilmer EN, Velat GJ, et al: Stromal cell-derived factor-1 promoted angiogenesis and inflammatory cell infiltration in aneurysm walls. J Neurosurg 120:73-86, 2014

28. Hudson JS, Hoyne DS, Hasan DM: Inflammation and human cerebral aneurysms: current and future treatment prospects. Future Neurol 8:10.2217/fnl.13.40, 2013 
29. Janiga G, Berg P, Sugiyama S, Kono K, Steinman DA: The Computational Fluid Dynamics Rupture Challenge 2013Phase I: prediction of rupture status in intracranial aneurysms. AJNR Am J Neuroradiol 36:530-536, 2015

30. Jansen IGH, Schneiders JJ, Potters WV, van Ooij P, van den Berg R, van Bavel E, et al: Generalized versus patientspecific inflow boundary conditions in computational fluid dynamics simulations of cerebral aneurysmal hemodynamics. AJNR Am J Neuroradiol 35:1543-1548, 2014

31. Jin D, Sheng J, Yang X, Gao B: Matrix metalloproteinases and tissue inhibitors of metalloproteinases expression in human cerebral ruptured and unruptured aneurysm. Surg Neurol 68 (Suppl 2):S11-S16, 2007

32. Kawaguchi T, Nishimura S, Kanamori M, Takazawa H, Omodaka S, Sato K, et al: Distinctive flow pattern of wall shear stress and oscillatory shear index: similarity and dissimilarity in ruptured and unruptured cerebral aneurysm blebs. J Neurosurg 117:774-780, 2012

33. Kleinloog R, de Mul N, Verweij BH, Post JA, Rinkel GJE, Ruigrok YM: Risk factors for intracranial aneurysm rupture: a systematic review. Neurosurgery 82:431-440, 2018

34. Kosierkiewicz TA, Factor SM, Dickson DW: Immunocytochemical studies of atherosclerotic lesions of cerebral berry aneurysms. J Neuropathol Exp Neurol 53:399-406, 1994

35. Kulcsár Z, Ugron A, Marosfői M, Berentei Z, Paál G, Szikora I: Hemodynamics of cerebral aneurysm initiation: the role of wall shear stress and spatial wall shear stress gradient. AJNR Am J Neuroradiol 32:587-594, 2011

36. Lawton MT, Vates GE: Subarachnoid hemorrhage. N Engl J Med 377:257-266, 2017

37. Malek AM, Alper SL, Izumo S: Hemodynamic shear stress and its role in atherosclerosis. JAMA 282:2035-2042, 1999

38. Matsushige T, Akiyama Y, Okazaki T, Shinagawa K, Ichinose N, Awai K, et al: Vascular wall imaging of unruptured cerebral aneurysms with a hybrid of opposite-contrast MR angiography. AJNR Am J Neuroradiol 36:1507-1511, 2015

39. Meng H, Swartz DD, Wang Z, Hoi Y, Kolega J, Metaxa EM, et al: A model system for mapping vascular responses to complex hemodynamics at arterial bifurcations in vivo. Neurosurgery 59:1094-1101, 2006

40. Meng H, Tutino VM, Xiang J, Siddiqui A: High WSS or low WSS? Complex interactions of hemodynamics with intracranial aneurysm initiation, growth, and rupture: toward a unifying hypothesis. AJNR Am J Neuroradiol 35:1254-1262, 2014

41. Meng H, Wang Z, Hoi Y, Gao L, Metaxa E, Swartz DD, et al: Complex hemodynamics at the apex of an arterial bifurcation induces vascular remodeling resembling cerebral aneurysm initiation. Stroke 38:1924-1931, 2007

42. Millán RD, Dempere-Marco L, Pozo JM, Cebral JR, Frangi AF: Morphological characterization of intracranial aneurysms using 3-D moment invariants. IEEE Trans Med Imaging 26:1270-1282, 2007

43. Miura Y, Ishida F, Umeda Y, Tanemura H, Suzuki H, Matsushima S, et al: Low wall shear stress is independently associated with the rupture status of middle cerebral artery aneurysms. Stroke 44:519-521, 2013

44. Morita A, Kirino T, Hashi K, Aoki N, Fukuhara S, Hashimoto N, et al: The natural course of unruptured cerebral aneurysms in a Japanese cohort. N Engl J Med 366:2474-2482, 2012

45. Nader-Sepahi A, Casimiro M, Sen J, Kitchen ND: Is aspect ratio a reliable predictor of intracranial aneurysm rupture? Neurosurgery 54:1343-1348, 2004

46. Nagahata S, Nagahata M, Obara M, Kondo R, Minagawa N, Sato S, et al: Wall enhancement of the intracranial aneurysms revealed by magnetic resonance vessel wall imaging using three-dimensional turbo spin-echo sequence with motionsensitized driven-equilibrium: a sign of ruptured aneurysm? Clin Neuroradiol 26:277-283, 2016
47. Nakagawa D, Cushing C, Nagahama Y, Allan L, Hasan D: Quantitative susceptibility mapping as a possible tool to radiographically diagnose sentinel headache associated with intracranial aneurysm: case report. World Neurosurg 103:954.e1-954.e4, 2017

48. Nixon AM, Gunel M, Sumpio BE: The critical role of hemodynamics in the development of cerebral vascular disease. $\mathbf{J}$ Neurosurg 112:1240-1253, 2010

49. Nussbaum ES, Defillo A, Zelensky A, Pulivarthi S, Nussbaum L: "Microbleeding" from intracranial aneurysms: local hemosiderin deposition identified during microsurgical treatment of unruptured intracranial aneurysms. Surg Neurol Int 5:28, 2014

50. Ollikainen E, Tulamo R, Lehti S, Lee-Rueckert M, Hernesniemi J, Niemelä M, et al: Smooth muscle cell foam cell formation, apolipoproteins, and ABCA1 in intracranial aneurysms: implications for lipid accumulation as a promoter of aneurysm wall rupture. J Neuropathol Exp Neurol 75:689-699, 2016

51. Omodaka S, Sugiyama S, Inoue T, Funamoto K, Fujimura M, Shimizu H, et al: Local hemodynamics at the rupture point of cerebral aneurysms determined by computational fluid dynamics analysis. Cerebrovasc Dis 34:121-129, 2012

52. Penn DL, Witte SR, Komotar RJ, Sander Connolly E Jr: The role of vascular remodeling and inflammation in the pathogenesis of intracranial aneurysms. J Clin Neurosci 21:28-32, 2014

53. Rosanwo O, Petz C, Prohaska S, Hege HC, Hotz I: Dual streamline seeding, in Eades P, Ertl T, Shen HW (eds): IEEE Pacific Visualization Symposium 2009. Piscataway, NJ: IEEE, 2009, pp 9-16

54. Sforza DM, Putman CM, Cebral JR: Hemodynamics of cerebral aneurysms. Annu Rev Fluid Mech 41:91-107, 2009

55. Shojima M, Oshima M, Takagi K, Torii R, Hayakawa M, Katada K, et al: Magnitude and role of wall shear stress on cerebral aneurysm: computational fluid dynamic study of 20 middle cerebral artery aneurysms. Stroke 35:2500-2505, 2004

56. Signorelli F, Gory B, Riva R, Labeyrie PE, Pelissou-Guyotat I, Turjman F: Hemodynamics, inflammation, vascular remodeling, and the development and rupture of intracranial aneurysms: a review. Neuroimmunol Neuroinflamm 2:59-67, 2015

57. Szajer J, Ho-Shon K: A comparison of 4D flow MRI-derived wall shear stress with computational fluid dynamics methods for intracranial aneurysms and carotid bifurcations - a review. Magn Reson Imaging 48:62-69, 2018

58. Taylor CA, Steinman DA: Image-based modeling of blood flow and vessel wall dynamics: applications, methods and future directions. Ann Biomed Eng 38:1188-1203, 2010

59. Traub O, Berk BC: Laminar shear stress: mechanisms by which endothelial cells transduce an atheroprotective force. Arterioscler Thromb Vasc Biol 18:677-685, 1998

60. Tremmel M, Dhar S, Levy EI, Mocco J, Meng H: Influence of intracranial aneurysm-to-parent vessel size ratio on hemodynamics and implication for rupture: results from a virtual experimental study. Neurosurgery 64:622-631, 2009

61. Ujiie H, Tachibana H, Hiramatsu O, Hazel AL, Matsumoto T, Ogasawara Y, et al: Effects of size and shape (aspect ratio) on the hemodynamics of saccular aneurysms: a possible index for surgical treatment of intracranial aneurysms. Neurosurgery 45:119-130, 1999

62. Vakil P, Ansari SA, Cantrell CG, Eddleman CS, Dehkordi FH, Vranic J, et al: Quantifying intracranial aneurysm wall permeability for risk assessment using dynamic contrastenhanced MRI: a pilot study. AJNR Am J Neuroradiol 36:953-959, 2015

63. Wasserman SM, Topper JN: Adaptation of the endothelium to fluid flow: in vitro analyses of gene expression and in vivo implications. Vasc Med 9:35-45, 2004 
64. Wiebers DO, Whisnant JP, Huston J III, Meissner I, Brown RD Jr, Piepgras DG, et al: Unruptured intracranial aneurysms: natural history, clinical outcome, and risks of surgical and endovascular treatment. Lancet 362:103-110, 2003

65. Wong GKC, Poon WS: Current status of computational fluid dynamics for cerebral aneurysms: the clinician's perspective. J Clin Neurosci 18:1285-1288, 2011

66. Xiang J, Natarajan SK, Tremmel M, Ma D, Mocco J, Hopkins LN, et al: Hemodynamic-morphologic discriminants for intracranial aneurysm rupture. Stroke 42:144-152, 2011

67. Xiang J, Tutino VM, Snyder KV, Meng H: CFD: computational fluid dynamics or confounding factor dissemination? The role of hemodynamics in intracranial aneurysm rupture risk assessment. AJNR Am J Neuroradiol 35:1849-1857, 2014

68. Zhang X, Yao ZQ, Karuna T, Duan CZ, Wang XM, Li XF, et al: Cerebral microbleeds could be independently associated with intracranial aneurysm rupture: a cross-sectional population-based study. World Neurosurg 115:e218-e225, 2018

69. Zhou G, Zhu Y, Yin Y, Su M, Li M: Association of wall shear stress with intracranial aneurysm rupture: systematic review and meta-analysis. Sci Rep 7:5331, 2017

\section{Disclosures}

The authors report no conflict of interest concerning the materials or methods used in this study or the findings specified in this paper.

\section{Author Contributions}

Conception and design: Park, Soldozy. Acquisition of data: Soldozy, Elsarrag, Chatrath, Costello. Drafting the article: Soldozy, Elsarrag, Chatrath. Critically revising the article: Park, Soldozy, Norat, Elsarrag, Chatrath, Costello, Kalani. Reviewed submitted version of manuscript: Park, Soldozy, Norat, Elsarrag, Chatrath, Costello, Sokolowski, Tvrdik. Approved the final version of the manuscript on behalf of all authors: Park. Administrative/technical/material support: Park, Norat. Study supervision: Park, Kalani.

\section{Correspondence}

Min S. Park: University of Virginia Health System, Charlottesville, VA.mp2tq@virginia.edu. 